POS $\quad \begin{aligned} & \text { PROCEEDINGS } \\ & \text { OF SCIENCE }\end{aligned}$

\title{
Energy-momentum tensor on the lattice: recent developments
}

\author{
Hiroshi Suzuki* \\ Department of Physics, Kyushu University, 744 Motooka, Nishi-ku, Fukuoka, 819-0395, Japan \\ E-mail: hsuzukiephys.kyushu-u.ac.jp
}

It is conceivable that the construction of the energy-momentum tensor (EMT) in lattice field theory enlarges our ability in lattice field theory and also deepens our understanding on EMT at the non-pertubative level. In this talk, I will review recent developments in this enterprise.

34th annual International Symposium on Lattice Field Theory

24-30 July 2016

University of Southampton, UK

${ }^{*}$ Speaker. 


\section{Introduction}

The energy-momentum tensor (EMT) is a fundamental observable in quantum field theory, being the Noether current associated with the translational invariance. It generates the Poincare transformations and the dilatation through the Ward-Takahashi (WT) relations. It thus carries information concerning physical quantities associated with those spacetime symmetries, such as the energy, momentum, pressure, stress, angular momentum, viscosity, specific heat, and renormalization group functions. It is also a source of gravity. Thus, we have enough motivation to compute EMT by employing the lattice regularization, a most well-established non-perturbative formulation of quantum field theory. It has been recognized that, however, the construction of EMT on the lattice is not straightforward because the lattice regularization explicitly breaks the translational invariance; roughly speaking the situation is similar to the chiral symmetry on the lattice. In the present talk, I will review recent developments made on the construction of EMT in lattice (gauge) field theory.

\section{EMT with the dimensional regularization}

First, let us note that the description of EMT in gauge theory is particularly simple if one adopts the dimensional regularization. This is because this regularization manifestly preserves the (vectorial) gauge symmetry and the translational invariance. One can then derive WT relations associated with the translational invariance straightforwardly. For simplicity, let us take the pure Yang-Mills theory in a $D$-dimensional Euclidean spacetime,

$$
S=\frac{1}{4 g_{0}^{2}} \int d^{D} x F_{\mu v}^{a}(x) F_{\mu v}^{a}(x)
$$

where $g_{0}$ is the bare gauge coupling and

$$
F_{\mu v}(x)=\partial_{\mu} A_{v}(x)-\partial_{v} A_{\mu}(x)+\left[A_{\mu}(x), A_{v}(x)\right]
$$

is the field strength. Under the infinitesimal variation,

$$
\delta_{\alpha} A_{\mu}(x)=\alpha_{v}(x) F_{v \mu}(x)
$$

which is a particular combination of the translation with a localized parameter $\alpha_{v}(x)$ and a gauge transformation, the action changes as

$$
\delta_{\alpha} S=-\int d^{D} x \alpha_{v}(x) \partial_{\mu} T_{\mu v}(x)
$$

where $T_{\mu v}(x)$ is EMT in the pure Yang-Mills theory:

$$
T_{\mu v}(x)=\frac{1}{g_{0}^{2}}\left[F_{\mu \rho}^{a}(x) F_{v \rho}^{a}(x)-\frac{1}{4} \delta_{\mu v} F_{\rho \sigma}^{a}(x) F_{\rho \sigma}^{a}(x)\right] .
$$


Thus, considering the change of variable of the form $([2.3)$ in the functional integral containing gauge invariant operators $\mathscr{O}(y)$ and $\mathscr{O}(z)$, we have ${ }^{1}$

$$
\begin{aligned}
\left\langle\partial_{\mu} T_{\mu v}(x) \mathscr{O}(y) \mathscr{O}(z)\right\rangle=- & \left\langle\delta(x-y) \partial_{v} \mathscr{O}(y) \mathscr{O}(z)+\mathscr{O}(y) \delta(x-z) \partial_{v} \mathscr{O}(z)\right\rangle \\
& +(\text { terms being vanishing under the integration over } x),
\end{aligned}
$$

(the last term can have the structure such as $\partial_{\nu} \delta(x-y)$ ). This WT relation shows that EMT generates the infinitesimal translation and, at the same time, the bare quantity $\partial_{\mu} T_{\mu \nu}(x)$ does not receive any multiplicative renormalization. In the pure Yang-Mills theory, there is no gauge invariant second-rank symmetric tensor of the mass dimension smaller than or equal to 4 whose total divergence identically vanishes; the only exception is a constant multiple of $\delta_{\mu v}$. Thus the absolute normalization of EMT is fixed by the physical requirement (2.6). The renormalized finite EMT is then obtained by subtracting a possibly divergent vacuum expectation value (VEV), ${ }^{2}$

$$
T_{\mu v}(x) \rightarrow T_{\mu v}(x)-\left\langle T_{\mu v}(x)\right\rangle .
$$

Thus the construction of EMT is particularly simple with the dimensional regularization. This is the reason why the dimensional regularization is extensively employed to study the issue of the trace or conformal anomaly in gauge theory [四], for example. The drawback of the dimensional regularization is of course that it is defined only within perturbation theory.

\section{EMT with the lattice regularization}

A general strategy for the construction of EMT in lattice field theory was developed in detail in Refs. [四, [3]. The strategy is quite analogous to the one for the chiral symmetry on the lattice studied in Ref. [四]. ${ }^{3}$

We start with a possible definition of a "would-be infinitesimal translation" on the link variables $U(x, \mu)$. Since the infinitesimal translation is in any case broken by the lattice structure, the definition is to a large extent arbitrary. Here, corresponding to Eq. ([2.3), we set

$$
\hat{\delta}_{\alpha} U(x, \mu)=\alpha_{v}(x) \hat{F}_{v \mu}(x) U(x, \mu) .
$$

In principle, we can use any lattice transcription for the field strength, $\hat{F}_{v \mu}(x)$. To make the transformation under the hypercubic transformation simple, however, it is convenient to adopt the clover definition for $\hat{F}_{v \mu}(x)$. We then consider the change of variable of the form (B.]) in the functional integral containing gauge invariant lattice operators $\hat{\mathscr{O}}(y)$ and $\hat{\mathscr{O}}(z)$. This time, we have $\left(\hat{\partial}_{\mu}\right.$ being a lattice derivative)

$$
\left\langle\left[\hat{\partial}_{\mu} \hat{T}_{\mu \nu}^{\text {naive }}(x)+\hat{X}_{v}(x)\right] \hat{\mathscr{O}}(y) \hat{\mathscr{O}}(z)\right\rangle=-\left\langle\frac{\delta}{\delta \alpha_{v}(x)} \hat{\delta}_{\alpha} \hat{\mathscr{O}}(y) \hat{\mathscr{O}}(z)+\hat{\mathscr{O}}(y) \frac{\delta}{\delta \alpha_{v}(x)} \hat{\delta}_{\alpha} \hat{\mathscr{O}}(z)\right\rangle .
$$

\footnotetext{
${ }^{1}$ Here, we implicitly assume the existence of the gauge-fixing and the Faddeev-Popov ghost terms. The variation of these terms, however, is BRS exact and can be neglected in correlation functions of gauge invariant operators.

${ }^{2}$ With the dimensional regularization, $T_{\mu \nu}$ will be finite even without such a subtraction; here we define the renormalized EMT by subtracting VEV to make expressions be consistent also with the lattice regularization.

${ }^{3}$ It is interesting to note that a computation of the trace anomaly of the Wilson fermion in a pioneering work []] yielded -15 times the conventional trace anomaly. Later, it was realized in Ref. []] that the reason for this discrepancy is that the translation WT relation is not imposed in Ref. [[]].
} 
In this expression, the naive lattice transcription of the classical EMT $\hat{T}_{\mu \nu}^{\text {naive }}(x)$ is defined by

$$
\hat{T}_{\mu v}^{\text {naive }}(x) \equiv \hat{T}_{\mu \nu}^{[6]}(x)+\hat{T}_{\mu v}^{[3]}(x)
$$

from

$$
\begin{aligned}
& \hat{T}_{\mu \nu}^{[6]}(x) \equiv \frac{1}{g_{0}^{2}}\left(1-\delta_{\mu \nu}\right) \sum_{\rho} \hat{F}_{\mu \rho}^{a}(x) \hat{F}_{v \rho}^{a}(x), \\
& \hat{T}_{\mu \nu}^{[3]}(x) \equiv \frac{1}{g_{0}^{2}} \delta_{\mu \nu}\left[\sum_{\rho} \hat{F}_{\mu \rho}^{a}(x) \hat{F}_{v \rho}^{a}(x)-\frac{1}{4} \sum_{\rho \sigma} \hat{F}_{\rho \sigma}^{a}(x) \hat{F}_{\rho \sigma}^{a}(x)\right] .
\end{aligned}
$$

These $\hat{T}_{\mu \nu}^{[6]}(x)$ and $\hat{T}_{\mu \nu}^{[3]}(x)$ transform as the sextet and the triplet representations under the hypercubic group, respectively; $\hat{T}_{\mu \nu}^{[6]}(x)$ possesses only off-diagonal components in $\mu$ and $v$, while $\hat{T}_{\mu \nu}^{[3]}(x)$ possesses only diagonal components and is traceless. Since $\hat{T}_{\mu \nu}^{\text {naive }}(x)$ coincides with EMT in the classical theory in the tree-level continuum limit, the breaking term $\hat{X}_{v}(x)$ in Eq. (B.2), which arises from the non-invariance of the lattice action under the would-be translation (B.D) with $\alpha_{v}=$ const., is $O(a)$ in the tree-level continuum limit. However, since $\hat{T}_{\mu \nu}^{\text {naive }}(x)$ is traceless $\sum_{\mu} \hat{T}_{\mu \mu}^{\text {naive }}(x)=0$ and does not reproduce the trace anomaly [四], $\hat{T}_{\mu \nu}^{\text {naive }}(x)$ cannot be the correct EMT in quantum theory.

In fact, though the radiative corrections, the breaking term $\hat{X}_{v}(x)$ can be $O\left(a^{0}\right)$ in correlation functions. Assuming lattice symmetries such as the hypercubic symmetry, one infers the expansion,

$$
\hat{X}_{v}(x)=\left(\frac{Z_{6}}{Z_{\delta}}-1\right) \hat{\partial}_{\mu} \hat{T}_{\mu v}^{[6]}(x)+\left(\frac{Z_{3}}{Z_{\delta}}-1\right) \hat{\partial}_{\mu} \hat{T}_{\mu v}^{[3]}(x)+\frac{Z_{1}}{Z_{\delta}} \hat{\partial}_{\mu} \hat{T}_{\mu v}^{[1]}(x)+\frac{1}{Z_{\delta}} \hat{R}_{v}(x),
$$

where $Z_{\alpha}(\alpha=6,3$, and 1$)$ and $Z_{\delta}$ are constants and the last term $\hat{R}_{v}(x)$ is $O(a)$ in correlation functions if it is separated from other operators in position space. In Eq. (3.6), the new combination

$$
\hat{T}_{\mu \nu}^{[1]}(x) \equiv \frac{1}{g_{0}^{2}} \delta_{\mu v} \sum_{\rho \sigma} \hat{F}_{\rho \sigma}^{a}(x) \hat{F}_{\rho \sigma}^{a}(x)
$$

transforms as the singlet representation under the hypercubic group; $\hat{T}_{\mu v}^{[1]}(x)$ possesses only diagonal components and is trace-ful.

Substituting the expansion (B.6) into the identity (B.2), we have

$$
\begin{aligned}
& \left\langle\hat{\partial}_{\mu}\left[Z_{6} \hat{T}_{\mu \nu}^{[6]}(x)+Z_{3} \hat{T}_{\mu \nu}^{[3]}(x)+Z_{1} \hat{T}_{\mu \nu}^{[1]}(x)\right] \hat{\mathscr{O}}(y) \hat{\mathscr{O}}(z)\right\rangle \\
& =-\left\langle Z_{\delta} \frac{\delta}{\delta \alpha_{v}(x)} \hat{\delta}_{\alpha} \hat{\mathscr{O}}(y) \hat{\mathscr{O}}(z)+\hat{\mathscr{O}}(y) Z_{\delta} \frac{\delta}{\delta \alpha_{v}(x)} \hat{\delta}_{\alpha} \hat{\mathscr{O}}(z)+\hat{R}_{v}(x) \hat{\mathscr{O}}(y) \hat{\mathscr{O}}(z)\right\rangle .
\end{aligned}
$$

The statement of the restoration of the translational invariance in the continuum limit is that one can choose the constant $Z_{\delta}$ as $a \rightarrow 0$ so that the right-hand side of the above expression becomes (recall Eq. (12.6))

$$
\begin{aligned}
\stackrel{a \rightarrow 0}{\rightarrow}-\langle & \left.\delta(x-y) \partial_{v} \mathscr{O}(y) \mathscr{O}(z)+\mathscr{O}(y) \delta(x-z) \partial_{v} \mathscr{O}(z)\right\rangle \\
& +(\text { terms being vanishing under the integration over } x)
\end{aligned}
$$


where $\mathscr{O}(y)$ and $\mathscr{O}(z)$ are operators in the continuum theory corresponding to $\hat{\mathscr{O}}(y)$ and $\hat{\mathscr{O}}(z)$, respectively. Assuming this is possible, the properly-normalized EMT which reproduces the correct WT relation in the continuum limit is given by

$$
\hat{T}_{\mu \nu}(x)=Z_{6} \hat{T}_{\mu \nu}^{[6]}(x)+Z_{3} \hat{T}_{\mu \nu}^{[3]}(x)+Z_{1}\left[\hat{T}_{\mu \nu}^{[1]}(x)-\left\langle\hat{T}_{\mu \nu}^{[1]}(x)\right\rangle\right]
$$

where we have subtracted the vacuum expectation value (only the trace-ful diagonal $\hat{T}_{\mu \nu}^{[1]}(x)$ can possess the vacuum expectation value). Thus, our mission is to determine the renormalization constants, $Z_{6}, Z_{3}$, and $Z_{1}$.

There are various strategies to determine the renormalization constants:

- Eqs. (B.8) and (B.9) reduce to the conservation law of EMT if we set $x \neq y$ and $x \neq z$. This determines the ratios $Z_{3} / Z_{6}$ and $Z_{1} / Z_{6}$. The overall normalization $Z_{6}$ in Eq. (B.TO) may be fixed by the rest energy $-\hat{T}_{00}$ of a hadronic state [ $[2$, , [3].

- Use a matching of the thermal expectation value of Eq. (B.10) with bulk thermodynamic quantities (the energy-density and the pressure) obtained by one of standard methods [ $[\mathbb{Q}, \mathbb{Z}]$. This determines $Z_{3}$ and $Z_{1}$ but tells nothing about $Z_{6}$, the coefficient of off-diagonal elements of EMT.

- Use physical normalization conditions implied by shifted boundary conditions; $Z_{6}$ can also

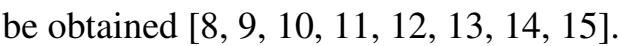

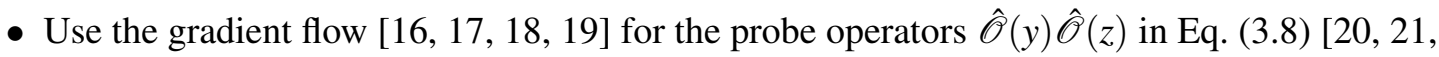
[22, [23].

In the present talk, I will review the last two recent ideas. In Sect. G, I will explain an alternative approach which does not rely on the representation (B.JU); it employs the gradient flow to construct EMT itself [24, 25], 26, 27, 28, [29, [3], B], 132, 133, 134, [3]].

\section{Shifted boundary conditions [8, Q, 10, 11, 12, 131, 14, 15]}

By employing boundary conditions with a spatial shift, one can derive physical normalization conditions which can be used to determine renormalization constants for EMT in Eq. (B.JU), especially $Z_{6}$ for the off-diagonal elements. For this, we consider the free energy density with a spatial shift vector $\boldsymbol{\xi}$ :

$$
f\left(L_{0}, \boldsymbol{\xi}\right)=-\frac{1}{V} \ln \operatorname{Tr}\left[e^{-L_{0}(H-i \boldsymbol{\xi} \cdot \boldsymbol{P})}\right],
$$

where $L_{0}$ denotes the temporal size of the system and $V$ is the spatial volume; $H$ and $\boldsymbol{P}$ are the Hamiltonian and the momentum operators, respectively. This shifted partition function can be expressed by the functional integral over a field $\phi\left(x_{0}, \boldsymbol{x}\right)$ with the shifted boundary condition, $\phi\left(L_{0}, \boldsymbol{x}\right)=\phi\left(0, \boldsymbol{x}-L_{0} \boldsymbol{\xi}\right)$. Then the derivative with respect to the $i$-th component of the shift vector, $\xi_{i}$, gives rise to the $i$-th momentum,

$$
\frac{\partial}{\partial \xi_{i}} f\left(L_{0}, \boldsymbol{\xi}\right)=-\frac{1}{V} i L_{0}\left\langle P_{i}\right\rangle_{\boldsymbol{\xi}}=-L_{0}\left\langle T_{0 i}\right\rangle_{\boldsymbol{\xi}}
$$


This condition can be used to determine $Z_{6}$ in Eq. (B.J) if the left-hand side of this relation can be computed; for an efficient method for this computation, see Ref. [14]].

We can also derive interesting relations by relating systems with shifted and un-shifted boundary conditions respectively by noting the underlying $S O(4)$ symmetry (in the continuum theory). For a shift vector of the form $\boldsymbol{\xi}=\left(\xi_{1}, 0,0\right)$, for example, the $S O(4)$ rotation

$$
R=\left(\begin{array}{cccc}
\gamma_{1} & \gamma_{1} \xi_{1} & 0 & 0 \\
-\gamma_{1} \xi_{1} & \gamma_{1} & 0 & 0 \\
0 & 0 & 1 & 0 \\
0 & 0 & 0 & 1
\end{array}\right), \quad \gamma_{1}=\frac{1}{\sqrt{1+\xi_{1}^{2}}}
$$

make the boundary condition un-shifted. Since EMT transforms as a tensor under the rotation, we have

$$
\left\langle T_{\mu v}\right\rangle_{\xi}=\sum_{\rho, \sigma} R_{\mu \rho}^{T} R_{v \sigma}^{T}\left\langle T_{\rho \sigma}\right\rangle
$$

where the expectation value in the right-hand side is defined with respect to the boundary condition without the shift. By considering this relation for $(\mu, v)=(0,0),(0,1)$, and $(1,1)$ and noting $\left\langle T_{01}\right\rangle=0$ in the thermodynamic limit (which is assumed here), we have

$$
\left\langle T_{0 i}\right\rangle_{\boldsymbol{\xi}}=\frac{\xi_{i}}{1-\xi_{i}^{2}}\left\langle T_{00}-T_{i i}\right\rangle_{\boldsymbol{\xi}}
$$

by eliminating the expectation values with the un-shifted boundary condition from the relation (4.4) in favor of the expectation values with the shifted one. This relation can be used to determine the ratio $Z_{3} / Z_{6}$.

One can also derive

$$
\frac{\partial}{\partial \xi_{i}}\left\langle\sum_{\mu} T_{\mu \mu}\right\rangle_{\boldsymbol{\xi}}=\frac{1}{\left(1+\boldsymbol{\xi}^{2}\right)^{2}} \frac{\partial}{\partial \xi_{i}}\left[\frac{\left(1+\boldsymbol{\xi}^{2}\right)^{3}}{\xi_{i}}\left\langle T_{0 i}\right\rangle_{\boldsymbol{\xi}}\right],
$$

which may be used to determine the ratio $Z_{1} / Z_{6}$.

On the basis of the renormalization constants $Z_{6}$ and $Z_{3} / Z_{6}$ obtained from the above relations, a very accurate entropy density of the $S U(3)$ pure Yang-Mills theory at finite temperature is calculated [15]]. See also Ref. [36] for updated results.

\section{The gradient flow is used for the probe operators [20, 21, 22, 23]}

Let us recall the would-be translation WT relation, Eq. (3.8). In the right-hand side of this equation, the operator $\hat{R}_{v}(x)$ can provide $O\left(a^{0}\right)$ contributions when it collides with other operators (the so-called contact term) although $\hat{R}_{v}(x)$ itself is $O(a)$ when it is separated from other operators in position space. This can happen, in particular, when we integrate the WT relation over the coordinate $x$; the point $x$ then collides with the points $y$ and $z$. Thus, it is not straightforward to isolate the terms being proportional to $Z_{\delta}$ in Eq. (B.8). As we will see below, however, the gradient

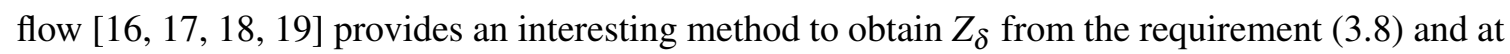
the same time $Z_{6}, Z_{3}$, and $Z_{1}$ from the WT relation. 
The gradient flow is a one-parameter evolution of the gauge field. For the gauge potential in the continuum theory $A_{\mu}(x)$, the evolution along the flow time $t$ is defined by

$$
\partial_{t} B_{\mu}(t, x)=D_{v} G_{v \mu}(t, x), \quad B_{\mu}(t=0, x)=A_{\mu}(x),
$$

where

$$
\begin{aligned}
G_{\mu v}(t, x) & =\partial_{\mu} B_{v}(t, x)-\partial_{v} B_{\mu}(t, x)+\left[B_{\mu}(t, x), B_{v}(t, x)\right], \\
D_{\mu} & =\partial_{\mu}+\left[B_{\mu}, \cdot\right],
\end{aligned}
$$

are the field strength of the flowed gauge field and the covariant derivative with respect to $B_{\mu}(t, x)$, respectively. I emphasize that the initial condition of the flow equation (I) is the bare gauge field. A surprising feature of the gradient flow, which was perturbatively proven in Ref. [एव] (see also Ref. [37]), is that any composite operator of the flowed gauge field for a positive flow time $t>0$ automatically becomes a renormalized finite operator; moreover it does not produce any new UV divergences even if other composite operators collide with it.

Thus let us suppose that we take certain composite operators composed from the flowed gauge field as the probe operators $\hat{\mathscr{O}}(y) \hat{\mathscr{O}}(z)$ in Eq. (B.8). For example, we may take

$$
\left.\hat{\mathscr{O}}(y) \equiv \hat{\partial}_{\rho} \hat{T}_{\rho \nu}^{[\alpha]}(y)\right|_{\text {flowed lattice gauge field at the flow time } t>0}, \quad \alpha=6,3,1, \quad \hat{\mathscr{O}}(z) \equiv 1 .
$$

Then because of the UV finiteness of the gradient flow, as $a \rightarrow 0$ the contribution of $\hat{R}_{v}(x)$ in Eq. (B.8) can be neglected even for $x=y$ and $x=z$ and we have ${ }^{4}$

$$
\begin{aligned}
& \left\langle\hat{\partial}_{\mu}\left[Z_{6} \hat{T}_{\mu \nu}^{[6]}(x)+Z_{3} \hat{T}_{\mu \nu}^{[3]}(x)+Z_{1} \hat{T}_{\mu \nu}^{[1]}(x)\right] \hat{\mathscr{O}}(y)\right\rangle=-\left\langle Z_{\delta} \frac{\delta}{\delta \alpha_{\nu}(x)} \hat{\delta}_{\alpha} \hat{\mathscr{O}}(y)\right\rangle+O(a) \\
& \stackrel{a \rightarrow 0}{\rightarrow}-\left\langle\delta(x-y) \partial_{\nu} \mathscr{O}(y)\right\rangle+(\text { terms being vanishing under the integration over } x) .
\end{aligned}
$$

Since we know the explicit form of $\frac{\delta}{\delta \alpha_{v}(x)} \hat{\delta}_{\alpha} \hat{\mathscr{O}}(y)$, the first equality for the prove operator (5.4) with $\alpha=6,3$, and 1 , provides a possible method to determine the ratios $Z_{6} / Z_{\delta}, Z_{3} / Z_{\delta}$, and $Z_{1} / Z_{\delta}$ for $a \rightarrow 0$. Also from the last requirement in the continuum limit, one may extract the renormalization constant $Z_{\delta}$ itself. For explicit results obtained by the above strategy, see Refs. [23] and [38]. For an application to the 3D $\lambda \phi^{4}$ theory, see Ref. [B]]

\section{Universal formula for EMT [24, 25, 26, 27, 28, 29, 30, 31, 32, 33, 34, 35]}

\subsection{Small flow time representation of EMT}

In this section, I explain an alternative approach [24, 26] to EMT on the lattice, which is based on the UV finiteness of composite operators constructed by the gradient flow (D.J) and the flow for the fermion field [40]:

$$
\begin{array}{ll}
\partial_{t} \chi(t, x)=\Delta \chi(t, x), & \chi(t=0, x)=\psi(x) \\
\partial_{t} \bar{\chi}(t, x)=\bar{\chi}(t, x) \overleftarrow{\Delta}, & \bar{\chi}(t=0, x)=\bar{\psi}(x)
\end{array}
$$

\footnotetext{
${ }^{4}$ Here, we adopt a boundary condition which breaks the translational invariance; then generally the right-hand side does not vanish.
} 
where, on fermion fields,

$$
\begin{array}{rlrl}
\Delta & =D_{\mu} D_{\mu}, & D_{\mu} & =\partial_{\mu}+B_{\mu} \\
\overleftarrow{\Delta} & \equiv \overleftarrow{D}_{\mu} \overleftarrow{D}_{\mu}, & \overleftarrow{D}_{\mu} \equiv \overleftarrow{\partial}_{\mu}-B_{\mu}
\end{array}
$$

This approach gives rise to a "universal formula" for EMT, which is rather different from the representation (B.10).

The approach can be developed for general vector-like gauge theories $\left(D_{\mu} \equiv \partial_{\mu}+A_{\mu}\right.$ for the fermion):

$$
S=\frac{1}{4 g_{0}^{2}} \int d^{D} x F_{\mu v}^{a}(x) F_{\mu v}^{a}(x)+\int d^{D} x \bar{\psi}(x)\left(\mathbb{D}+m_{0}\right) \psi(x),
$$

where $m_{0}$ is the bare fermion mass parameter. As in Sect. \, assuming the dimensional regularization, by considering an infinitesimal variation of integration variables in the functional integral of the form,

$$
\delta_{\alpha} A_{\mu}(x)=\alpha_{v}(x) F_{v \mu}(x), \quad \delta_{\alpha} \psi(x)=\alpha_{\mu}(x) D_{\mu} \psi(x),
$$

we have EMT which satisfies the translation WT identity. The explicit form is given by [U]

$$
\begin{aligned}
T_{\mu \nu}(x)=\frac{1}{g_{0}^{2}} & {\left[F_{\mu \rho}^{a}(x) F_{v \rho}^{a}(x)-\frac{1}{4} \delta_{\mu v} F_{\rho \sigma}^{a}(x) F_{\rho \sigma}^{a}(x)\right] } \\
& +\frac{1}{4} \bar{\psi}(x)\left(\gamma_{\mu} \overleftrightarrow{D}_{v}+\gamma_{v} \overleftrightarrow{D}_{\mu}\right) \psi(x)-\delta_{\mu \nu} \bar{\psi}(x)\left(\frac{1}{2} \overleftrightarrow{\mathbb{D}}+m_{0}\right) \psi(x)
\end{aligned}
$$

where

$$
\overleftrightarrow{D}_{\mu} \equiv D_{\mu}-\overleftarrow{D}_{\mu}, \quad \overleftarrow{D}_{\mu} \equiv \overleftarrow{\partial}_{\mu}-A_{\mu}
$$

Note however that the expression (6.]) is meaningful only under the perturbative dimensional regularization; it cannot be employed with the lattice regularization as it stands.

At this point we recall that any local composite operator composed from the flowed gauge field $B_{\mu}(t, x)$ in Eq. (5.J) and the flowed fermion fields $\chi(t, x)$ in Eq. (6. (ل) and $\bar{\chi}(t, x)$ in Eq. (6.2) for $t>0$ automatically becomes a renormalized finite operator, if the flowed fermion fields are multiplicatively renormalized [며, 40] (see also Ref. [B]]):

$$
\chi_{R}(t, x)=Z_{\chi}^{1 / 2} \chi(t, x), \quad \bar{\chi}_{R}(t, x)=Z_{\chi}^{1 / 2} \bar{\chi}(t, x) .
$$

These wave function renormalizations may be avoided by using the following "ringed variables" [[26]: ${ }^{5}$

$$
\begin{aligned}
& \stackrel{\chi}{\chi}(t, x) \equiv \sqrt{\frac{-2 \operatorname{dim}(R) N_{f}}{(4 \pi)^{2} t^{2}\langle\bar{\chi}(t, x) \overleftrightarrow{D} \chi(t, x)\rangle}} \chi(t, x), \\
& \stackrel{\circ}{\chi}(t, x) \equiv \sqrt{\frac{-2 \operatorname{dim}(R) N_{f}}{(4 \pi)^{2} t^{2}\langle\bar{\chi}(t, x) \overleftrightarrow{\mathbb{D}} \chi(t, x)\rangle}} \bar{\chi}(t, x),
\end{aligned}
$$

where $\operatorname{dim}(R)$ denotes the dimension of the gauge representation $R$ to which the fermion is belonging, because the wave function renormalization constant $Z_{\chi}$ is canceled out in $\stackrel{\circ}{\chi}(t, x)$ and in $\stackrel{\circ}{\chi}(t, x)$.

\footnotetext{
${ }^{5}$ In what follows, we assume that all fermion masses are degenerate; $N_{f}$ denotes the number of degenerated flavors.
} 
Thus any composite of $B_{\mu}(t, x), \grave{\chi}(t, x)$, and $\stackrel{\circ}{\chi}(t, x)$ for $t>0$ is a renormalized finite operator. Such a renormalized finite operator should possess a meaning being independent of the regularization, if the renormalization conditions are taken equally. In what follows, we construct a certain local combination of $B_{\mu}(t, x), \dot{\chi}(t, x)$, and $\stackrel{\circ}{\chi}(t, x)$ which coincides with Eq. (6.7) if one uses the dimensional regularization. This composite operator of flowed fields thus becomes EMT under the dimensional regularization and on the other hand it possesses a meaning being independent of the regularization. Thus, assuming the existence of EMT in the non-perturbative level, this combination must be $E M T$. This is our strategy. From this reasoning it is clear that our expression for EMT is universal in the sense that it should hold with any regularization, not only with the lattice regularization.

The problem is however how to obtain such a combination of flowed fields which reduces to Eq. (6.7) under the dimensional regularization. The relationship between the original fields at the vanishing flow time and the flowed fields at $t>0$ is quite non-trivial because the flow equations are non-linear in field variables.

The relationship however can be tractable at least in one situation, the small flow time limit $t \rightarrow$ 0 . This is the so-called small flow time expansion [ए9] which infers that for $t \rightarrow 0$ a local composite operator of flowed fields at $t>0$ can be represented by an asymptotic series of local composite operators of un-flowed fields with increasing mass dimensions. For example, in the pure YangMills theory, we have (here, $\mathbb{1}$ denotes the identity operator)

$$
\begin{aligned}
G_{\mu \rho}^{a}(t, x) G_{v \rho}^{a}(t, x) \stackrel{t \rightarrow 0}{\sim}\langle & \left.G_{\mu \rho}^{a}(t, x) G_{v \rho}^{a}(t, x)\right\rangle \mathbb{1} \\
& +\zeta_{11}(t)\left[F_{\mu \rho}^{a}(x) F_{v \rho}^{a}(x)-\left\langle F_{\mu \rho}^{a}(x) F_{v \rho}^{a}(x)\right\rangle\right] \\
& \quad+\zeta_{12}(t)\left[\delta_{\mu v} F_{\rho \sigma}^{a}(x) F_{\rho \sigma}^{a}(x)-\left\langle\delta_{\mu v} F_{\rho \sigma}^{a}(x) F_{\rho \sigma}^{a}(x)\right\rangle\right]+O(t) .
\end{aligned}
$$

Since the flow time possesses the mass dimension -2 , the $O(t)$ term in the above expansion starts from local operators of the mass dimension 6 . The expansion coefficients such as $\zeta_{11}(t)$ and $\zeta_{12}(t)$ in the dimensional regularization for $t \rightarrow 0$, moreover, can be worked out by perturbation theory if the theory is asymptotically free [ㅍ]. See also Ref. [4] for an efficient method for the computation. Thus, considering the $t \rightarrow 0$ limit to neglect the $O(t)$ term in Eq. (6.12), one can express the first line of Eq. (6.7) (in the pure Yang-Mills theory) in terms of the flowed gauge field by inverting the above relation with respect to $F_{\mu \rho}^{a}(x) F_{v \rho}^{a}(x)-\mathrm{VEV}$ (this is always possible within perturbation theory).

Carrying out the above procedure, ${ }^{6}$ we have [24, 26]

$$
\begin{gathered}
T_{\mu v}(x)=\lim _{t \rightarrow 0}\left\{c_{1}(t)\left[\tilde{\mathscr{O}}_{1 \mu v}(t, x)-\frac{1}{4} \tilde{\mathscr{O}}_{2 \mu v}(t, x)\right]\right. \\
+c_{2}(t)\left[\tilde{\mathscr{O}}_{2 \mu v}(t, x)-\left\langle\tilde{\mathscr{O}}_{2 \mu v}(t, x)\right\rangle\right] \\
+c_{3}(t)\left[\tilde{\mathscr{O}}_{3 \mu v}(t, x)-2 \tilde{\mathscr{O}}_{4 \mu v}(t, x)-\left\langle\tilde{\mathscr{O}}_{3 \mu v}(t, x)-2 \tilde{\mathscr{O}}_{4 \mu v}(t, x)\right\rangle\right] \\
+c_{4}(t)\left[\tilde{\mathscr{O}}_{4 \mu v}(t, x)-\left\langle\tilde{\mathscr{O}}_{4 \mu v}(t, x)\right\rangle\right] \\
\left.+c_{5}(t)\left[\tilde{\mathscr{O}}_{5 \mu v}(t, x)-\left\langle\tilde{\mathscr{O}}_{5 \mu v}(t, x)\right\rangle\right]\right\}
\end{gathered}
$$

\footnotetext{
${ }^{6}$ This idea has been examined analytically by using solvable models [ [27, [2, [0]].
} 
where

$$
\begin{aligned}
& \tilde{\mathscr{O}}_{1 \mu v}(t, x) \equiv G_{\mu \rho}^{a}(t, x) G_{v \rho}^{a}(t, x), \\
& \tilde{\mathscr{O}}_{2 \mu v}(t, x) \equiv \delta_{\mu v} G_{\rho \sigma}^{a}(t, x) G_{\rho \sigma}^{a}(t, x), \\
& \tilde{\mathscr{O}}_{3 \mu v}(t, x) \equiv \stackrel{\circ}{\chi}(t, x)\left(\gamma_{\mu} \overleftrightarrow{D}_{v}+\gamma_{v} \overleftrightarrow{D}_{\mu}\right) \dot{\chi}(t, x) \\
& \tilde{\mathscr{O}}_{4 \mu v}(t, x) \equiv \delta_{\mu v} \stackrel{\circ}{\bar{\chi}}(t, x) \overleftrightarrow{\mathbb{D}} \stackrel{\chi}{\chi}(t, x), \\
& \tilde{\mathscr{O}}_{5 \mu v}(t, x) \equiv \delta_{\mu v} m \stackrel{\circ}{\bar{\chi}}(t, x) \dot{\chi}(t, x) \text {. }
\end{aligned}
$$

The coefficients are given by, through the one-loop perturbation theory, ${ }^{7}$

$$
\begin{aligned}
& c_{1}(t)=\frac{1}{\bar{g}(1 / \sqrt{8 t})^{2}}-b_{0} \ln \pi-\frac{1}{(4 \pi)^{2}}\left[\frac{7}{3} C_{2}(G)-\frac{3}{2} T(R) N_{f}\right], \\
& c_{2}(t)=\frac{1}{8} \frac{1}{(4 \pi)^{2}}\left[\frac{11}{3} C_{2}(G)+\frac{11}{3} T(R) N_{f}\right], \\
& c_{3}(t)=\frac{1}{4}\left\{1+\frac{\bar{g}(1 / \sqrt{8 t})^{2}}{(4 \pi)^{2}} C_{2}(R)\left[\frac{3}{2}+\ln (432)\right]\right\}, \\
& c_{4}(t)=\frac{1}{8} d_{0} \bar{g}(1 / \sqrt{8 t})^{2}, \\
& c_{5}(t)=-\frac{\bar{m}(1 / \sqrt{8 t})}{m}\left\{1+\frac{\bar{g}(1 / \sqrt{8 t})^{2}}{(4 \pi)^{2}} C_{2}(R)\left[3 \ln \pi+\frac{7}{2}+\ln (432)\right]\right\},
\end{aligned}
$$

and

$$
b_{0}=\frac{1}{(4 \pi)^{2}}\left[\frac{11}{3} C_{2}(G)-\frac{4}{3} T(R) N_{f}\right], \quad d_{0}=\frac{1}{(4 \pi)^{2}} 6 C_{2}(R),
$$

where $\bar{g}(q)$ and $\bar{m}(q)$ are the running gauge coupling and the running mass in the MS scheme, respectively. The validity of the formula (6.13) for the lattice regularization has been examined numerically for the thermodynamics of the $S U$ (3) pure Yang-Mills theory [25, [28, B], B2], B3] ] and of the $N_{f}=2+1$ QCD [B3, [35] with very encouraging results. For the $S U(3)$ pure Yang-Mills theory, two-point correlation functions of EMT has been computed [42, 43] which even indicate the conservation law of EMT.

Our universal formula (6.13) with the universal coefficients (6.19)-(6.23) is thought to be valid for any sound regularization, including the lattice regularization with any sensible lattice discretization. Moreover, from the dimensional counting, it is conceivable that the expressions are valid even in curved space ${ }^{8}$ if straightforward suitable modifications by using the curved (euclidean) metric are made. For example, the flow equation (5.]) will be replaced by

$$
\partial_{t} B_{\mu}(t, x)=g^{v \rho}(x) \mathscr{D}_{\nu} G_{\rho \mu}(t, x), \quad B_{\mu}(t=0, x)=A_{\mu}(x),
$$

where $g^{v \rho}(x)$ and $\mathscr{D}_{v}$ are the curved space metric and the covariant derivative, respectively. Thus, if we have a lattice regularization on a curved manifold with which the general coordinate invariance

\footnotetext{
${ }^{7}$ The quadratic Casimirs are defined from anti-hermitian group generators by $\operatorname{tr}_{R}\left(T^{a} T^{b}\right)=-T(R) \delta^{a b}, T^{a} T^{a}=$ $-C_{2}(R) 1$, and $f^{a c d} f^{b c d}=C_{2}(G) \delta^{a b}$, where $\left[T^{a}, T^{b}\right]=f^{a b c} T^{c}$.

${ }^{8}$ If the scalar field exists, we will have additional terms in EMT which contain the scalar curvature.
} 
can be restored in the continuum limit (see Refs. [44, 45] for a recent attempt), we will be able to use our universal formula to study physics related to EMT on a curved manifold. ${ }^{9}$

Now, although our formula is expected to be universal, this universality holds only after the renormalization and sending the cutoff to infinity. With the lattice regularization, the universality hence holds only in the continuum limit; we have to first set $a \rightarrow 0$ while $t$ is kept fixed in physical unit and then take the $t \rightarrow 0$ limit as Eq. (6.53). ${ }^{10}$ In actual numerical simulations with a finite lattice spacing $a$, we thus have a natural window for a sensible range of $t$ :

$$
a \ll \sqrt{8 t} \ll \frac{1}{\Lambda}
$$

where $\Lambda$ is a mass scale of the low energy physics (such as the hadron mass, temperature, volume etc.). This picture comes from the fact that the diffusion length of the flow equations is $\sim \sqrt{8 t}$. Thus, when only finite lattice spacings are available, we cannot simply set $t \rightarrow 0$ and then it is not a priori obvious whether our universal formula (6.53) is practically useful. The numerical experiments so far indicate that the formula is of real use, but still we have to understand and reduce the systematic error associated with the $t \rightarrow 0$ extrapolation. In the next two subsections, I present some observations related to this issue.

\section{2 $O(t)$ correction in the continuum limit}

To make the extrapolation to $t \rightarrow 0$ for Eq. (6.13) from very small but finite $t$, it will be quite helpful (or crucial) to have some idea on the nature of the $O(t)$ term we have neglected from the small flow time expansion to derive Eq. (6.13), such as the last term of Eq. (6.12).

From the general form of the small flow time expansion in Ref. [प्प], such an $O(t)$ term has the structure,

$$
t \sum_{i} c_{i}(t ; g, m ; \mu)\left\{\mathscr{O}_{i}\right\}_{R}(x)=t \sum_{i} c_{i}(t ; g, m ; \mu) \sum_{j}\left(Z^{-1}\right)_{i j} \mathscr{O}_{j}(x),
$$

where $c_{i}(t ; g, m ; \mu)$ are dimensionless coefficients and $\left\{\mathscr{O}_{i}\right\}_{R}(x)$ are dimension 6 renormalized operators ( $\mu$ denotes the renormalization scale). In the right-hand side, we have re-expressed the renormalized operators in terms of bare operators $\mathscr{O}_{j}(x)$ by using the renormalization constants. Since we are considering a composite operator of flowed fields which are some combination of bare fields, the derivative of Eq. (6.27) with respect to the renormalization scale $\mu$ vanishes. Thus

$$
\left(\mu \frac{\partial}{\partial \mu}\right)_{0}\left[\sum_{i} c_{i}(t ; g, m ; \mu)\left(Z^{-1}\right)_{i j}\right]=0
$$

where the subscript 0 implies that the derivative is taken with all bare quantities are kept fixed and, in terms of conventional renormalization group functions $\beta \equiv\left(\mu \frac{\partial}{\partial \mu}\right)_{0} g$ and $\gamma_{m} \equiv-\left(\mu \frac{\partial}{\partial \mu}\right)_{0} \ln m$, we have

$$
\left(\mu \frac{\partial}{\partial \mu}+\beta \frac{\partial}{\partial g}-\gamma_{m} m \frac{\partial}{\partial m}-\gamma_{i}\right) c_{i}(t ; g, m ; \mu)=0, \quad \text { no sum over } i .
$$

\footnotetext{
${ }^{9}$ I would like to thank George T. Fleming for a discussion on this possibility.

${ }^{10}$ In Ref. [B4], this double limit is literally taken and very encouraging results are obtained.
} 
Here, we have introduced the matrix $\gamma_{i j} \equiv-\sum_{k}\left(\mu \frac{\partial}{\partial \mu}\right)_{0}\left(Z^{-1}\right)_{j k} Z_{k i}$ and assumed an appropriate operator basis with which $\gamma_{i j}$ is diagonal, $\gamma_{i j}=\gamma_{i} \delta_{i j}$. Then, by analyzing the $t \rightarrow 0$ behavior of the solution to this equation, we conclude

$$
c_{i}(t ; g, m ; \mu)=C_{i} \bar{g}(1 / \sqrt{8 t})^{2\left[1+\gamma_{i 0} /\left(2 b_{0}\right)\right]}\left[1+O\left(\bar{g}(1 / \sqrt{8 t})^{2}\right)\right]+O(t),
$$

where $\gamma_{i 0}$ denotes the one-loop coefficient of the anomalous dimension $\gamma_{i}(g), \gamma_{i}=\gamma_{i 0} g^{2}+O\left(g^{4}\right)$ (the one-loop coefficient in $\beta, b_{0}$, is given by Eq. (6.24)). Thus, for $t \rightarrow 0$, the $O(t)$ term (6.27) is dominated by a dimension 6 operator with the smallest one-loop anomalous dimension $\gamma_{i 0}$. Although it seems not easy to enumerate anomalous dimensions of all dimension 6 operators which appear in the small flow time expansion relevant for EMT, the above observation still might be useful in making a realizable $t \rightarrow 0$ extrapolation in future numerical simulations.

\section{3 $O(a)$ correction for a fixed $t$}

Quite often, I was asked the relationship between the conventional strategy for EMT on the lattice in Eq. (B.Jd) and the universal formula (6.13) and, closely related to this point, why the one-loop perturbative coefficients in Eqs. (6.J)-(6.23) are expected or seem to work in numerical simulations, although the one-loop perturbative renormalization constants have been known to be generally insufficient for presently-accessible lattice parameters (for the axial vector current, for example). ${ }^{11}$

I think that the point is quite related to an important property of the gradient flow and deserves a closer look. Let us recall the representation of a lattice composite operator in Symanzik's effective theory [46] (Ref. [47] is a very nice exposition):

$$
Z_{\mathscr{O}}(a) \hat{\mathscr{O}}(x)=\mathscr{O}(x)+a \mathscr{O}^{\prime}(x)+a^{2} \mathscr{O}^{\prime \prime}(x)+\cdots,
$$

where $\hat{\mathscr{O}}(x)$ is a bare composite operator in lattice theory with a spacing $a$ and $Z_{\mathscr{O}}(a)$ is a renormalization constant for $\hat{\mathscr{O}}(x)$; in the right-hand side, $\mathscr{O}(x), \mathscr{O}^{\prime}(x), \ldots$, are all renormalized composite operators in Symanzik's effective theory. We see that only when the renormalization constant $Z_{\mathscr{O}}(a)$ is taken into account, the approach of the lattice operator $Z_{\mathscr{O}}(a) \hat{\mathscr{O}}(x)$ to the continuum one $\mathscr{O}(x)$ becomes linear in $a .{ }^{12}$ The lower order lattice perturbation theory for $Z_{\mathscr{O}}(a)$ would not be realizable because of the "tadpole dominance" [48]] and a non-perturbative determination of $Z_{\mathscr{O}}(a)$ would be required. Now, if we apply the same idea to a composite operator of flowed lattice fields, we would have

$$
\hat{\mathscr{O}}(t, x)=\mathscr{O}(t, x)+a \mathscr{O}^{\prime}(t, x)+a^{2} \mathscr{O}^{\prime \prime}(t, x)+\cdots,
$$

which is a relation holding in the effective theory considered in Ref. [49] for the gradient flow in lattice theory. Here, the crucial difference from Eq. (6.3I) is that the left-hand side is already a renormalized quantity and we do not need a renormalization constant such as $Z_{\mathscr{O}}(a)$ in Eq. (6.31).

Thus, from the representation (6.32), we see that, for a fixed flow time $t>0$, the approach of the lattice operator $\hat{\mathscr{O}}(t, x)$ to the continuum counterpart $\mathscr{O}(t, x)$ is linear in a (up to logarithmic

\footnotetext{
${ }^{11}$ From colleagues including, Shinya Aoki, Shoji Hashimoto, Naruhito Ishizuka, Yoshio Kikukawa, Yoshinobu Kuramashi, and Yusuke Taniguchi, to whom I would like to thank for discussions.

${ }^{12}$ In the pure Yang-Mills theory, the approach would be linear in $a^{2}$.
} 
factors). ${ }^{13}$ This expected $a \rightarrow 0$ behavior of composite operators of flowed fields can be found, for example, in Figs. 3 and 4 of Ref. [34]. Then, to reduce the slope to the $a \rightarrow 0$ extrapolation, the improvement ideas [49, 50, 5]] will be very useful.

The one-loop perturbative matching coefficients in Eqs. (6.19)-(6.23), which tell how we should take the $t \rightarrow 0$ extrapolation, are on the other hand obtained by the continuum perturbation theory; this does not suffer from the tadpole dominance.

\section{Conclusion}

There have been rapid developments recently on the construction of EMT in lattice field theory, an old but important problem, with encouraging results. So far, tests and/or applications of new ideas are limited mostly to bulk thermodynamics (i.e., one-point functions of EMT). Considering the vast amount of potential applications, such as the spin/momentum structure of hadrons, (quasi)conformal field theory, large anomalous dimensions, gravity, etc. and that they are mainly related to correlation functions of EMT, we expect much to be explored.

On the small flow-time approach presented in the last section, it is interesting that one can have a closed universal expression for EMT. Applications to the bulk thermodynamics show encouraging results. In this approach, as a "by-product" of the smearing effect of the flow, there is a tendency that the noise in correlation functions is suppressed. Still, we need to further understand and reduce the systematic error associated with the $t \rightarrow 0$ extrapolation.

\section{Acknowledgments}

I would like to thank Francesco Capponi, Leonardo Giusti, Agostino Patella, Michele Pepe, and Antonio Rago for providing me useful information on their works. I am also grateful to collaborators in the FlowQCD Collaboration and the WHOT-QCD Collaboration for enjoyable collaborations. The work of H. S. is supported in part by JSPS Grants-in-Aid for Scientific Research Grant Number 16H03982.

\section{References}

[1] J. C. Collins, A. Duncan and S. D. Joglekar, Phys. Rev. D 16, 438 (1977). doi:10.1103/PhysRevD.16.438

[2] S. Caracciolo, G. Curci, P. Menotti and A. Pelissetto, Annals Phys. 197, 119 (1990). doi:10.1016/0003-4916(90)90203-Z

[3] S. Caracciolo, P. Menotti and A. Pelissetto, Nucl. Phys. B 375, 195 (1992). doi:10.1016/0550-3213(92)90339-D

[4] M. Bochicchio, L. Maiani, G. Martinelli, G. C. Rossi and M. Testa, Nucl. Phys. B 262, 331 (1985). doi:10.1016/0550-3213(85)90290-1

[5] K. Fujikawa, Z. Phys. C 25, 179 (1984). doi:10.1007/BF01557477

\footnotetext{
${ }^{13}$ Again, in the pure Yang-Mills theory, the approach would be linear in $a^{2}$.
} 
[6] H. B. Meyer, Phys. Rev. D 76, 101701 (2007) doi:10.1103/PhysRevD.76.101701 [arXiv:0704.1801 [hep-lat]].

[7] K. Huebner, F. Karsch and C. Pica, Phys. Rev. D 78, 094501 (2008) doi:10.1103/PhysRevD.78.094501 [arXiv:0808.1127 [hep-lat]].

[8] L. Giusti and H. B. Meyer, Phys. Rev. Lett. 106, 131601 (2011) doi:10.1103/PhysRevLett.106.131601 [arXiv:1011.2727 [hep-lat]].

[9] L. Giusti and H. B. Meyer, JHEP 1111, 087 (2011) doi:10.1007/JHEP11(2011)087 [arXiv:1110.3136 [hep-lat]].

[10] L. Giusti and H. B. Meyer, JHEP 1301, 140 (2013) doi:10.1007/JHEP01(2013)140 [arXiv:1211.6669 [hep-lat]].

[11] D. Robaina and H. B. Meyer, PoS LATTICE 2013, 323 (2014) [arXiv:1310.6075 [hep-lat]].

[12] L. Giusti and M. Pepe, Phys. Rev. Lett. 113, 031601 (2014) doi:10.1103/PhysRevLett.113.031601 [arXiv:1403.0360 [hep-lat]].

[13] M. Pepe and L. Giusti, PoS LATTICE 2014, 322 (2014) [arXiv:1410.8371 [hep-lat]].

[14] L. Giusti and M. Pepe, Phys. Rev. D 91, 114504 (2015) doi:10.1103/PhysRevD.91.114504 [arXiv:1503.07042 [hep-lat]].

[15] L. Giusti and M. Pepe, PoS LATTICE 2015, 211 (2016) [arXiv:1511.03710 [hep-lat]].

[16] R. Narayanan and H. Neuberger, JHEP 0603, 064 (2006) doi:10.1088/1126-6708/2006/03/064 [hep-th/0601210].

[17] M. Lüscher, Commun. Math. Phys. 293, 899 (2010) doi:10.1007/s00220-009-0953-7 [arXiv:0907.5491 [hep-lat]].

[18] M. Lüscher, JHEP 1008, 071 (2010) Erratum: [JHEP 1403, 092 (2014)] doi:10.1007/JHEP08(2010)071, 10.1007/JHEP03(2014)092 [arXiv:1006.4518 [hep-lat]].

[19] M. Lüscher and P. Weisz, JHEP 1102, 051 (2011) doi:10.1007/JHEP02(2011)051 [arXiv:1101.0963 [hep-th]].

[20] L. Del Debbio, A. Patella and A. Rago, JHEP 1311, 212 (2013) doi:10.1007/JHEP11(2013)212 [arXiv:1306.1173 [hep-th]].

[21] A. Patella, L. Del Debbio and A. Rago, PoS LATTICE 2013, 324 (2014).

[22] F. Capponi, A. Rago, L. Del Debbio, S. Ehret and R. Pellegrini, PoS LATTICE 2015, 306 (2016) [arXiv:1512.02851 [hep-lat]].

[23] F. Capponi, L. Del Debbio, A. Patella and A. Rago, PoS LATTICE 2015, 302 (2016) [arXiv:1512.04374 [hep-lat]].

[24] H. Suzuki, PTEP 2013, $083 B 03$ (2013) Erratum: [PTEP 2015, 079201 (2015)] doi:10.1093/ptep/ptt059, 10.1093/ptep/ptv094 [arXiv:1304.0533 [hep-lat]].

[25] M. Asakawa et al. [FlowQCD Collaboration], Phys. Rev. D 90, no. 1, 011501 (2014) Erratum: [Phys. Rev. D 92, no. 5, 059902 (2015)] doi:10.1103/PhysRevD.90.011501, 10.1103/PhysRevD.92.059902 [arXiv:1312.7492 [hep-lat]].

[26] H. Makino and H. Suzuki, PTEP 2014, 063 B02 (2014) Erratum: [PTEP 2015, 079202 (2015)] doi:10.1093/ptep/ptu070, 10.1093/ptep/ptv095 [arXiv:1403.4772 [hep-lat]]. 
[27] H. Makino and H. Suzuki, PTEP 2015, no. 3, 033 B08 (2015) doi:10.1093/ptep/ptv028 [arXiv:1410.7538 [hep-lat]].

[28] M. Kitazawa, M. Asakawa, T. Hatsuda, T. Iritani, E. Itou and H. Suzuki, PoS LATTICE 2014, 022 (2014) [arXiv:1412.4508 [hep-lat]].

[29] H. Makino, F. Sugino and H. Suzuki, PTEP 2015, no. 4, 043B07 (2015) doi:10.1093/ptep/ptv044 [arXiv:1412.8218 [hep-lat]].

[30] H. Suzuki, PTEP 2015, no. 4, 043B04 (2015) doi:10.1093/ptep/ptv036 [arXiv:1501.04371 [hep-lat]].

[31] E. Itou, H. Suzuki, Y. Taniguchi and T. Umeda, PoS LATTICE 2015, 303 (2016) [arXiv:1511.03009 [hep-lat]].

[32] M. Kitazawa, M. Asakawa, T. Hatsuda, T. Iritani, E. Itou and H. Suzuki, PoS LATTICE 2015, 162 (2016) [arXiv:1511.05235 [hep-lat]].

[33] Y. Taniguchi, S. Ejiri, R. Iwami, K. Kanaya, M. Kitazawa, H. Suzuki, T. Umeda and N. Wakabayashi, arXiv:1609.01417 [hep-lat].

[34] M. Kitazawa, T. Iritani, M. Asakawa, T. Hatsuda and H. Suzuki, arXiv:1610.07810 [hep-lat], to appear in Phys. Rev. D.

[35] K. Kanaya, S. Ejiri, R. Iwami, M. Kitazawa, H. Suzuki, Y. Taniguchi, T. Umeda and N. Wakabayashi, arXiv:1610.09518 [hep-lat].

[36] L. Giusti and M. Pepe, arXiv:1612.02337 [hep-lat].

[37] K. Hieda, H. Makino and H. Suzuki, arXiv:1604.06200 [hep-lat].

[38] F. Capponi, A. Patella, L. Del Debbio, and A. Rago, in these proceedings.

[39] F. Capponi, L. Del Debbio, S. Ehret, R. Pellegrini, A. Portelli and A. Rago, arXiv:1612.07721 [hep-lat].

[40] M. Lüscher, JHEP 1304, 123 (2013) doi:10.1007/JHEP04(2013)123 [arXiv:1302.5246 [hep-lat]].

[41] H. Suzuki, PTEP 2015, no. 10, 103B03 (2015) doi:10.1093/ptep/ptv139 [arXiv:1507.02360 [hep-lat]].

[42] M. Kitazawa, "QCD thermodynamics from Gradient Flow," talk delivered at The 13th International workshop on QCD in extreme conditions, http://conf.ccnu.edu.cn/ xqcd2015/

[43] M. Kitazawa et al. [FlowQCD Collaboration], in preparation.

[44] R. C. Brower, G. T. Fleming, A. D. Gasbarro, T. G. Raben, C. I. Tan and E. S. Weinberg, arXiv: 1610.08587 [hep-lat].

[45] R. C. Brower, G. T. Fleming, E. S. Weinberg, A. D. Gasbarro, T. G. Raben, and C. I. Tan, in these proceedings.

[46] K. Symanzik, Nucl. Phys. B 226, 187 (1983). doi:10.1016/0550-3213(83)90468-6

[47] P. Weisz, arXiv:1004.3462 [hep-lat].

[48] G. P. Lepage and P. B. Mackenzie, Phys. Rev. D 48, 2250 (1993) doi:10.1103/PhysRevD.48.2250 [hep-lat/9209022].

[49] A. Ramos and S. Sint, Eur. Phys. J. C 76, no. 1, 15 (2016) doi:10.1140/epjc/s10052-015-3831-9 [arXiv:1508.05552 [hep-lat]].

[50] Z. Fodor, K. Holland, J. Kuti, S. Mondal, D. Nogradi and C. H. Wong, JHEP 1409, 018 (2014) doi:10.1007/JHEP09(2014)018 [arXiv:1406.0827 [hep-lat]].

[51] N. Kamata and S. Sasaki, arXiv:1609.07115 [hep-lat]. 